\title{
Scepticisme et occultisme : le paranormal à l'œuvre dans Spirite de Théophile Gautier
}

\author{
Emir Delic \\ Université d'Ottawa
}

Ce qu'il y a d'admirable dans le fantastique, c'est qu'il n'y a plus de fantastique : il n'y a plus que le réel. Breton, 2005, p. 25

En art, une chose fausse peut être très vraie, et une chose vraie très fausse; tout dépend de l'exécution. Gautier, 1883, p. 45-46

Si l'on devait, en quelques coups de pinceau, esquisser un dessin qui rende compte des facettes multiples de Théophile 
Gautier, l'on pourrait sans aucun doute se rapporter à l'ouvrage d'Anne Ubersfeld sur la quatrième de couverture duquel on lit : "Romantique Gautier», "Sensuel Gautier», "Troublant Gautier », « Moderne Gautier » (1992). Cette galerie de portraits est certainement riche, mais il y aurait lieu de se demander si l'on pourrait (devrait?) y ajouter « Spirite Gautier».

Certes, Gautier ne fait pas exception parmi les écrivains du XIX ${ }^{\mathrm{e}}$ siècle dont la plupart se montrent, à des degrés divers, intrigués, voire enchantés, par la vogue des sciences occultes déclenchée surtout par la « découverte » du magnétisme animal en 1774 (Ellenberger, 1970, p.59). En effet, quoique discréditées par une commission d'enquête mandatée par Louis XVI en 1784, les théories de Franz Anton Mesmer ne cesseront d'attirer des adeptes fervents qui, en les modifiant, en assurent la pérennité au siècle suivant. Dans ce contexte, mentionnons notamment la contribution du marquis de Puységur qui, déjà à partir de 1785, propose l'idée du sommeil magnétique, idée qui fera rage dans la première moitié du XIXe siècle (ibid., p. 65-67, 70-72).

Ainsi, Gautier, tout comme Honoré de Balzac, Alexandre Dumas, Victor Hugo, Charles Nodier et George Sand, pour ne nommer que ceux-là, assiste à des séances où les somnambules font preuve de leurs talents extraordinaires (voir Edelman, 1995, p. 51-54, et Prévost, 2003, 2006). Que l'on considère les séances de magnétisme chez la vicomtesse Poillouë de SaintMars auxquelles Gautier assiste dès 1843 (voir Ricard, 1843, p. 92-93, 97-98) ${ }^{1}$, ou la séance de « magnétisme musical » qui a

\footnotetext{
${ }^{1}$ La vicomtesse, dont le nom de plume est comtesse Dash, atteste également la présence de Gautier (1886, t. 6, p. 73-74; cité par Lefebvre, 1993, p. 294).
} 
lieu chez Hugo et est rapportée par Gautier dans un article paru dans La Presse le 7 septembre 1847 (Montandon et Saminadayar-Perrin, 2003, p.281), ou encore les visites fréquentes de Gautier, dans les années 1850, chez Delphine de Girardin, qui rassemble autour d'elle tout un cercle d'adeptes de l'occultisme (Lefebvre, 1993, p. 293-294), on ne saurait nier la fascination qu'exercent le magnétisme et le somnambulisme sur Gautier. À plus forte raison quand on considère cette déclaration dans un article daté du 1er décembre 1838 et intitulé «Tronquette la Somnambule»: «Le magnétisme animal est un fait désormais acquis à la science et dont il n'est pas plus permis de douter que du galvanisme et de l'électricité.»(1858, t. 1, p. 201). Si Gautier adopte donc une attitude explicite à l'égard du magnétisme, il n'en est pas de même pour le spiritisme, mouvement qui, ayant pris naissance aux États-Unis en 1848 avec les sœurs Fox et avec l'apparition subséquente des "tables frappantes », connaît un immense succès en France à partir de 1853 (Edelman, 1995, p. 75-76).

Si nous nous proposons d'explorer ici l'attitude ambiguë de Gautier à l'égard du spiritisme, une question s'impose de prime abord: pourquoi l'opinion de Gautier serait-elle à ce propos plus importante que, disons, celle de Balzac ou celle d'Hugo? En quête de réponses à cette question, on ne manquera sûrement pas de souligner la renommée dont jouit Gautier comme critique littéraire et artistique, travaillant notamment pour LaPresse (1836-1855) et pour Le Moniteur universel (1855-1868) (Tortonese, 1995a). Sa réputation est effectivement telle qu'Allan Kardec, qui deviendra le théoricien principal du spiritisme, lui envoie en 1857 une copie de son ouvrage Le Livre des esprits en lui écrivant : 
Monsieur [...] Le point de vue nouveau, éminemment sérieux et moral dans lequel la doctrine des Esprits est envisagée, ajoute à l'intérêt qui s'attache à cette question à la fois d'avenir et d'actualité; et à laquelle, je crois, vous n'êtes pas étranger. [...] L'approbation d'un homme comme vous, Monsieur, me serait infiniment précieuse si j'étais assez heureux pour l'obtenir [...]. (Cité par Edelman, 1995, p. 110-111)

À la notoriété de Gautier comme critique littéraire et artistique se joint une autre raison importante pour laquelle Kardec sollicite son approbation, raison que l'on peut déceler dans les propos suivants de Kardec, tirés du Livre des médiums :

Le doute concernant l'existence des Esprits a pour cause première l'ignorance de leur véritable nature. On se les figure généralement comme des êtres à part dans la création, et dont la nécessité n'est pas démontrée. Beaucoup ne les connaissent que par les contes fantastiques dont ils ont été bercés, à peu près comme on connaît l'histoire par les romans; sans chercher si ces contes, dégagés des accessoires ridicules, reposent sur un fond de vérité, le côté absurde seul les frappe [...]. (1863, p. 1)

Si Kardec tente de faire de Gautier un partisan du spiritisme, c'est aussi parce que Gautier est un écrivain qui s'est adonné plus longtemps et plus fréquemment que d'autres pensons à Balzac, à Maupassant ou à Nodier — à la littérature fantastique (Eigeldinger, 1981, p. 17).

Afin d'explorer ces liens entre la littérature fantastique et les sciences occultes, notre étude se penchera sur la dernière œuvre fantastique de Gautier, qui est justement intitulée Spirite (1865) et qui est la seule, à part son premier conte, La Cafetière (1831; voir Gautier, 1981), à être qualifiée par l'auteur, par le truchement du sous-titre, de fantastique. Dans un premier temps, il s'agira d'examiner les manifestations et la nature du paranormal dans Spirite pour ensuite aborder la réception de 
l'œuvre, la conception particulière du fantastique de Gautier et son attitude à l'égard du paranormal.

\section{Scepticisme, spiritisme et swedenborgisme}

La nouvelle fantastique Spirite raconte l'histoire de l'esprit de Lavinia d'Aufideni, une jeune femme noble, qui après sa mort, ou mieux, après son passage dans le monde surnaturel, retourne dans le monde naturel sous la forme d'un esprit ou d'un ange afin de dévoiler son amour ineffable à Guy de Malivert, un jeune homme, noble lui aussi, qui ne connaissait pas Spirite lors de son séjour terrestre. C'est de cette transgression entre monde et «extra-monde» (p.337) que découlent les manifestations du paranormal qui se développent de manière progressive tout au long de la narration. Le premier événement explicitement paranormal a trait au spiritisme et se produit lorsque Malivert, pour excuser son absence à une soirée mondaine, essaie d'écrire un billet à Madame d'Ymbercourt, jeune veuve qui se voit déjà comme sa future épouse, mais dont Malivert ne partage aucunement la passion (p. 322-323). Ne sachant pas comment formuler son mot d'excuse, Malivert, après quelques instants d'hésitation, trouve sous ses yeux une missive très directe, voire impolie, qu'il a rédigée sans en avoir eu conscience (p.324-326). Le jeune homme, tentant de s'expliquer cette bizarrerie, avance comme raisons la folie et le somnambulisme (p.326). Comme ces états de conscience altérés constituent des justifications peu convaincantes et que Malivert, en regardant le billet, s'aperçoit que son écriture est également modifiée (p. 326), il continue la recherche d'une explication : 
Comment diable cette bizarre transformation a-t-elle pu se faire? Je n'ai cependant ni fumé d'opium, ni mangé de haschisch, et ce ne sont pas les deux ou trois verres de vin de Bordeaux que j'ai bus qui peuvent m'avoir porté à la tête. [...] Si j'étais un peu superstitieux, il ne tiendrait qu'à moi de voir là dedans un avertissement du ciel au lieu d'une distraction inqualifiable. (p. 326)

De toute évidence, ni l'opium, ni le haschisch, ni l'alcool ne se présentent comme des explications satisfaisantes pour éclaircir la façon dont s'est produite la « lettre qui s'était écrite pour ainsi dire toute seule»(p. 329)2. Cet incident insolite pourrait sans doute demeurer une "distraction inqualifiable", s'il n'était pas suivi d'un soupir que Malivert entend ou croit entendre au moment où il quitte son atelier-salon pour se rendre chez Madame d'Ymbercourt. Cette deuxième apparition explicite du surnaturel agite davantage l'esprit du protagoniste qui, en route pour la maison de Madame d'Ymbercourt, s'évertue de trouver des raisons rationnelles aux événements qu'il vient de vivre (p. 326-230). Bien qu'« [i]l se [dise] tout ce que les sceptiques allèguent de raisons naturelles pour expliquer l'incompréhensible» (p. 329), Malivert, par «un instinct secret», "[sent] que ce faible gémissement partait d'une âme et n'était pas un bruit vague de la matière » (p. 329). Par conséquent, il finira par conclure qu'il s'agit «[d']une intervention qu'il fallait bien qualifier de surnaturelle jusqu'à ce que l'analyse l'eût expliquée ou lui eût donné un autre titre » (p. 329-330). On le voit, les bizarreries laissent déjà planer un doute concernant leurs origines.

\footnotetext{
2 L'évocation de l'opium et du haschisch n'est pas gratuite. Gautier a publié deux contes, La Pipe d'opium (1838) et Le Club des hachichins (1846), où il relate les expériences qu'il a faites sous l'influence de ces drogues hallucinogènes. Voir Gautier (1981).
} 
Malivert a beau chercher une qualification autre que « surnaturelle » aux phénomènes singuliers dont il est témoin, il n'en trouvera aucune. C'est que, lors de la prochaine irruption du surnaturel, le soupir, qui - on s'en sera douté - est celui de Spirite, devient parole. Ainsi, le lendemain du premier incident étrange, Spirite chuchote " N'entrez pas » à l'oreille de Malivert qui est sur le point de sonner à la porte de Madame d'Ymbercourt (p.342). Tandis que cet «événement extranaturel » appelle encore une fois le protagoniste à avancer des justifications logiques, à savoir la folie et l'hallucination, dans la suite du récit, il en arrive à ne pas pouvoir nier l'existence de «puissances occultes dont il [sent] vaguement la présence autour de lui » (p. 351). Si Malivert, qui ne croit pas dans "les rêveries des magnétiseurs, des tables tournantes et des esprits frappeurs » (p. 351), est de plus en plus disposé à accepter la présence du surnaturel, c'est surtout parce que le personnage du baron de Féroë, «un voyant» (p. 431), deviendra « [son] mystagogue et [son] initiateur au surnaturel » (p. 356, 432). C'est lui qui confirme à Malivert qu'un esprit s'applique à communiquer avec lui et qui l'avise de la manière d'entrer en contact avec le monde invisible (p. 355-359) :

D'après ce que lui avait dit le baron de Féroë au club [...], Malivert rassembla toutes les puissances de son être, et formula intérieurement le désir d'entrer en communication plus directe avec l'esprit mystérieux qu'il pressentait autour de lui [...]. Cela fait, Malivert, qui était dans l'atelier-salon où il se trouvait au début de cette histoire, se mit à regarder et à écouter avec une attention extrême. Il ne vit et n'entendit d'abord rien, et cependant les objets qui meublaient cette pièce [...] lui paraissaient avoir pris des aspects étranges et qu'ils n'avaient pas d'ordinaire. Les lumières et les ombres projetées par la lampe leur prêtaient une vie fantastique [...]. (p. 361) 
Le miroir [de Venise] [...] exerçait sur lui une sorte de fascination et absorbait invinciblement son regard. [...] La tache lumineuse du miroir commençait à se dessiner d'une façon plus distincte et à se déteindre de couleurs légères, immatérielles pour ainsi dire, et qui auraient fait paraître terreux les tons de la plus fraîche palette. [...] L'image se condensait de plus en plus sans atteindre pourtant la précision grossière de la réalité, et Guy de Malivert put enfin voir [...] une tête de jeune femme, ou plutôt de jeune fille, d'une beauté dont la beauté mortelle n'est que l'ombre. (p. 362-363)

De ces passages, il ressort clairement que Malivert, suivant les conseils de son initiateur à l'extra-monde, s'ouvre à la possibilité de l'existence du surnaturel. De ce fait, Spirite se sait interpelée et elle apparaît dans le miroir.

Si nous venons de mettre l'accent sur les premières pages de la narration, c'est parce qu'il s'y dessine le schéma qui caractérise tout surgissement du paranormal ou bien toute rencontre du naturel et du surnaturel dans Spirite. En ce sens, à chaque prise de contact avec l'extra-monde, le doute de Malivert quant à la nature surnaturelle des phénomènes se dissipe jusqu'à ce que, peu après l'apparition de Spirite dans le miroir, il en vienne à l'étape suivante: « [I]l se livrait sans réserve à l'étrangeté de la situation, ne discutant rien, admettant tout et décidé de trouver le surnaturel naturel " (p. 365). On peut donc affirmer que plus l'apparition de Spirite est accusée, plus Malivert est attentif à la présence du surnaturel et moins il en questionne la positivité. Cette dynamique est bien résumée, en filigrane, par le baron de Féroë quand il avertit Malivert de ne pas chercher à « expliquer l'inexplicable », ce qui fermerait les portes de l'extra-monde (p. 433). En outre, à y regarder de près, Spirite se trouve déjà aux côtés de Malivert même avant que n'ait lieu aucun 
événement étrange qui trahirait sa présence. Ainsi, la raison pour laquelle, au tout début du récit, Malivert est « heureux sans qu'il lui [soit] arrivé aucun bonheur» (p.317) et qu' « [a]utour de lui tout [est] repos, bien-être, silence discret, quiétude intime » (p. 318) réside dans le fait que Spirite voltige invisiblement autour de lui (p. 367) ${ }^{3}$. Il s'ensuit que la présence de Spirite se signale d'abord sous un mode invisible pour ensuite se manifester de manières audible (le soupir mystérieux) et visible (l’apparition dans le miroir).

Cette progression des manifestations du surnaturel se précise dans le reste du récit. En effet, à l'apparition de Spirite dans le miroir suit, le lendemain, une manifestation dans le Bois de Boulogne, où Spirite se présente dans un traîneau. On passe donc d'une apparition à deux dimensions à une apparition à trois dimensions. Il importe cependant de noter que, dans la scène du traîneau, Spirite, qui se dissimule dans la calèche, n'est toujours pas tout à fait visible (p. 376-377). En revanche, une autre apparition tridimensionnelle de Spirite ressurgit ce même soir dans l'atelier-salon de Malivert, qui aperçoit une main se profiler sur le fond du tapis (p. 383-384). Et, deux jours plus tard, ce n'est pas seulement une main, mais Spirite qui se manifeste entièrement. Dans cette dernière apparition, Spirite revêt une sorte de forme corporelle, mais elle demeure toujours un «fantôme aérien» (p. 443), un être impalpable, sa matérialisation étant juste assez dense pour être perceptible pour Malivert (p.437). C'est sous cette forme aérienne que Spirite se présente à Malivert jusqu'à la fin du récit.

3 Spirite précise dans la suite du récit: " [P]our mieux vous garder, je répandais dans votre logis un vague enchantement qui vous le faisait aimer » (p. 430). 
Cela dit, il importe maintenant d'insister sur deux manifestations clefs du surnaturel dans Spirite. Il s'agit tout particulièrement de scènes où Spirite, en faisant de sa main les signes de l'écriture, fait comprendre à Malivert qu'il doit « être [son] propre médium » (p. 384) :

Une autre âme, ou du moins une autre pensée se substituait à la sienne et commandait à ces serviteurs qui, pour agir, attendent l'ordre du maître inconnu. Les nerfs de ses doigts tressaillirent et commencèrent à exécuter des mouvements dont il n'avait pas la conscience, et le bec de la plume se mit à courir sur le papier, traçant des signes rapides avec l'écriture de Guy légèrement modifiée par une impulsion étrangère. (p. 385)

Les fruits de cette écriture médiumnique, laquelle occupe Malivert pendant deux soirées consécutives, sont deux « dictées » au moyen desquelles Spirite renseigne Malivert sur son existence dans le monde et dans l'extra-monde, et lui avoue son amour (p.385-431). Bien que le terme de médium soit employé pour la première fois dans le passage que nous venons de citer, on ne peut s'empêcher de songer au premier billet adressé à Madame d'Ymbercourt qui, lui aussi, a été écrit de manière inconsciente. En effet, si l'on envisage la narration de Spirite dans une perspective globale, on peut affirmer que Malivert passe par plusieurs types de médiumnité, tels que définis par Allan Kardec dans Le Livre des médiums. Dans cet ouvrage, Kardec désigne par le mot médium « [t]oute personne qui ressent à un degré quelconque l'influence des Esprits » (1863, p. 195). Si l'on adopte cette définition, et pour ne relever que les facultés médiumniques qui nous semblent les plus importantes dans Spirite, on constate que Malivert est présenté au début du récit comme un "médium novice ", c'est-à-dire un médium «dont les facultés ne sont point encore complétement développées et qui [manque] de l'expérience nécessaire [sic] » 
(ibid., p. 230-231) et comme un " médium sensitif », car, comme on l'a remarqué, il est « [susceptible] de ressentir la présence des Esprits par une impression générale ou locale, vague ou matérielle » (ibid., p. 225).

$\mathrm{Au}$ fur et à mesure que le récit avance, on découvre chez ce personnage d'autres facultés médiumniques. Il se révèle, par exemple, comme un "médium nocturne», «qui n'[obtient] certains effets physiques que dans l'obscurité » (ibid., p.226), ainsi que l'illustre bien la scène de la première apparition de Spirite dans le miroir de Venise. Parmi tous les types médiumniques, le «médium écrivain mécanique», "dont la main reçoit une impulsion involontaire et qui n'[a] aucune conscience de ce qu'[il écrit]» (ibid., p. 230), et le «médium polygraphe », «dont l'écriture change avec l'Esprit qui se communique » (ibid.), en constituent les plus importants, étant donné la place occupée par les dictées de Spirite. Au demeurant, Spirite exprime bien cette gradation relativement à la mise en contact entre le monde et l'extra-monde quand elle déclare à Malivert : «Il fallait peu à peu vous préparer à mon apparition et me mettre mystérieusement en rapport avec vous; entre un esprit et un vivant non initié les communications sont difficiles. » (p.430) Il en découle que les différentes facultés médiumniques que Malivert acquiert suivant le cheminement de la narration correspondent nettement non seulement aux manifestations de plus en plus accusées de Spirite, mais aussi à l'effritement progressif du scepticisme que manifestait Malivert au début du récit.

Curieusement, les voies de communication de Spirite et de Malivert ne se limitent pas à l'écriture mécanique. Tout efficace qu'elle soit, celle-ci se voit dépassée par d'autres 
moyens de communication, ce qui nous amène à considérer une seconde science occulte représentée dans Spirite à égalité avec le spiritisme: le swedenborgisme. Remarquons à ce sujet d'abord qu'en ce qui concerne la communication entre Malivert et Spirite, les idées du baron de Féroë, qui est, d'ailleurs, « un Suédois, [un] compatriote de Swedenborg» et « swedenborgiste » (p. 334, 352), se heurtent à celles de Kardec. Le guide de Malivert affirme à ce propos que l'écriture médiumnique fait partie des «moyens lents et grossiers de communication » et que les deux amants se passeront bientôt de cette manière de communiquer l'un avec l'autre (p. 432) C'est là une circonstance qui se concrétise peu après, puisque Spirite réussira à parler à Malivert « d'une voix qui n'[est] pas formulée en paroles, mais que Guy [entend] dans le fond de son cœur» (p. 440). La communication d'âme qui s'établit ainsi entre les deux protagonistes n'est pourtant qu'un élément relevant du swedenborgisme. D'autres éléments clefs de cette doctrine se révèlent, par exemple, lorsque Spirite décrit les sphères qu'elle habite :

Des mots humains ne peuvent rendre la sensation d'une âme qui, délivrée de sa prison corporelle, passe de cette vie dans l'autre, du temps dans l'éternité et du fini dans l'infini. [...] Débarrassée de cette argile soumise aux lois de la pesanteur, qui m'alourdissait naguère encore, je m'élançais avec une alacrité folle dans l'insondable éther. Les distances n'existaient plus pour moi, et mon simple désir me rendait présente où je voulais être. Je traçais de grands cercles d'un vol plus rapide que la lumière à travers l'azur vague des espaces, comme pour

\footnotetext{
4 Par contre, Kardec affirme ceci : «De tous les moyens de communication, l'écriture manuelle est le plus simple, le plus commode, et surtout le plus complet. » (1863, p. 214)
} 
prendre possession de l'immensité, me croisant avec des essaims d'âmes et d'esprits. (p. 427)

L'impossibilité d'exprimer l'extra-monde en termes humains, la pesanteur du corps empêchant le mouvement de l'âme, et l'espace atemporel "où l'on peut ce que l'on veut" (p. 368), ce sont là d'importants principes de la doctrine de Swedenborg auxquels le récit fait allusion à plusieurs reprises 5 . L'extrait que nous venons de citer met aussi en évidence la nature phosphorique de l'au-delà, laquelle a partie liée, comme le suggèrent d'autres scènes dans Spirite, avec la luminosité intense qui caractérise l'être angélique. Que l'on songe à ce propos, par exemple, à la scène où Malivert est contraint de se détourner de Spirite lorsque, dans un instant d'extase, elle est sur le point de revêtir sa forme angélique et que, de ce fait, il émane d'elle une lumière resplendissante (p. 439)6.

Le dernier point important qu'il faut noter au sujet du swedenborgisme touche à la notion du mariage céleste. Tout comme dans le cas des divers aspects du spiritisme et de la médiumnité, cet élément crucial de la doctrine de Swedenborg

\footnotetext{
${ }^{5} \mathrm{Au}$ sujet de la pesanteur de la chair, le baron de Féroë déclare à Malivert lors de leur premier dîner : "Il n'y aurait pas besoin [...] de vous adresser le reproche que l'ange blanc fit un jour à Swedenborg: "Tu manges trop!" Vous êtes ce soir d'une sobriété exemplaire, et l'on croirait que vous essayez de vous spiritualiser par le jeûne.» (p.355) Pour ce qui est de la nature insaisissable de l'au-delà, Spirite dit à Malivert au début de la première dictée : "Il faut d'abord que vous connaissiez l'être indéfinissable pour vous qui s'est glissé dans votre existence. Quelle que soit votre pénétration, vous ne pourriez parvenir à démêler sa vraie nature [...]. » (p. 385) Voir aussi Spirite p. $367,383,432-433,444$.

6 Spirite déclare: « [J]'oubliais que tu es encore retenu dans ta prison terrestre et que tes yeux ne peuvent supporter le plus faible rayon de la vraie lumière. Plus tard, je me montrerai à toi, telle que je suis, dans la sphère où tu me suivras. Maintenant l'ombre de ma forme mortelle suffit à te manifester ma présence, et tu peux me contempler ainsi sans péril. » (p. 439)
} 
est annoncé d'entrée de jeu. Retournons alors au début du récit et portons encore notre attention sur la missive destinée à Madame d'Ymbercourt et écrite par Malivert sous l'influence de Spirite : «Vous êtes assez belle et entourée d'assez d'adorateurs pour qu'on puisse vous dire sans vous offenser qu'on ne vous aime pas. [...] À quoi bon continuer des relations qui finiraient par engager deux âmes si peu faites l'une pour l'autre et les lier dans un malheur éternel? » (p. 325-326) Nous apprenons donc, dès les premières pages de la narration, que l'âme de Malivert n'est pas faite pour celle de Madame d'Ymbercourt, mais plutôt pour celle de Spirite (voir aussi p. 388, 429). Voilà pourquoi Spirite intervient dans la vie terrestre de Malivert, et ce, non seulement en essayant de l'éloigner de Madame d'Ymbercourt, mais aussi en le détournant de tout engagement terrestre susceptible de séparer son âme de la sienne (p. 368). Il est évident qu'à l'aide de ses apparitions, Spirite réussit à faire voir et à faire sentir à Malivert que leurs âmes sont prédestinées l'une à l'autre. L'idée d'un tel destin de l'âme, de même que la possibilité de voies de communication dépassant la médiumnité, va à l'encontre de la doctrine de $\operatorname{Kardec}^{7}$. Il n'empêche qu'à la fin de la narration, les âmes de Malivert et de Spirite s'unissent et, conformément aux théories de Swedenborg (voir 1973, p. 277-278), forment un ange d'amour'.

\footnotetext{
7 Kardec affirme dans Le Livre des esprits : « Il faut [...] rejeter cette idée que deux Esprits créés l'un pour l'autre doivent un jour fatalement se réunir dans l'éternité, après avoir été séparés pendant un laps de temps plus au moins long. » (1867, p. 141).

8 Bien que la doctrine de Swedenborg soit, de manière globale, représentée fidèlement dans Spirite, il est à peu près certain qu'en ce qui concerne le swedenborgisme, Gautier se soit principalement inspiré de Balzac. Cette hypothèse semble d'autant plus plausible que, contrairement à ce qui est
} 
Les manifestations du paranormal dans Spirite concernent, somme toute, à la fois le spiritisme et le swedenborgisme, sciences occultes dont les principes sont, dans l'ensemble, fidèlement représentés. De plus, le paranormal, dans cette nouvelle de Gautier, s'affirme de manière progressive, ce qui a pour effet d'estomper le scepticisme initial de Malivert - et peut-être aussi de tel ou tel lecteur - à l'égard de l'occultisme. La question se pose maintenant de savoir quelle a été la réception de l'œuvre.

\section{Le paranormal à l'œuvre}

Étant donné la présence simultanée du spiritisme et du swedenborgisme ainsi que la démarche narrative qui assure une introduction graduelle du paranormal dans Spirite, la réception ambigüe qu'a connue cette dernière nouvelle de Gautier est peu étonnante. Écoutons dans ce contexte d'abord l'auteur qui, dans une célèbre lettre à Carlotta Grisi, écrit en décembre 1865 :

Je vous dirai que Spirite, en dehors de son effet littéraire a produit un effet magique des plus étranges, tout le monde des tables tournantes et des esprits frappeurs a été profondément remué. On s'imagine que je suis un initié, et que je sais le grand secret. J'ai reçu dans ce sens les lettres les plus bizarrement folles, entre'autres une venant de Grenoble, où l'inconnu qui signe P S prétend qu'il est Malivert, qu'il a une Spirite et me demande par quelle vision j'ai pu découvrir son histoire [...]. L'un [des journaux allemands] prétend que Spirite a été

indiqué dans Spirite, Swedenborg n'a pas écrit d'ouvrage intitulé Mariages de l'autre vie, ouvrage que lit le baron de Féroë à la fin du récit lorsqu'il contemple le mariage céleste de Malivert et de Spirite (p. 472-473). À ce sujet, se référer notamment à Tortonese (1995b, p. 1707-1709) et à Lefebvre (1996). 
commandée par le gouvernement pour occuper les esprits et les détourner de l'annexion de la Belgique. [...] Enfin il paraît que j'ai touché sans le savoir à l'inconnu, au mystère, au grand secret, à quelque chose de terrible et de profond, car tout le monde s'émeut [...]. [L]a presse de Vienne prétend que je ne suis moi-même qu'un médium [sic]. (Gautier, 1985, t. 9, p. 147149; c'est nous qui soulignons)

Les observations suivantes de Judith Gautier, la fille de l'auteur, répondent en écho à celles de son père :

[L]e succès littéraire de Spirite fut doublé d'un autre succès, sur lequel on ne comptait pas : les médiums, les magnétiseurs, les partisans des tables tournantes, ceux qui interrogent les esprits frappeurs, les swedenborgiens, s'émurent et adressèrent à Théophile Gautier les lettres les plus singulières et les plus folles. (1903, p. 324)

Deux articles publiés dans La Revue spirite et rédigés, fort probablement, par Allan Kardec lui-même ne reflètent pas tout à fait l'engouement public que suggèrent les propos de Gautier et de sa fille. Alors que le premier de ces textes, daté de décembre 1865, exprime quelques réserves sur la sincérité de Gautier $^{9}$, celles-ci se volatilisent presque complètement dans le deuxième texte qui paraît en mars 1866 :

La vérité est dans le fond des idées et des pensées, qui sont essentiellement spirites et rendues avec une délicatesse et une grâce charmantes, bien plus que dans les faits, dont la possibilité est parfois contestable. Quoique roman, cet ouvrage n'en a pas moins une grande importance par le nom de l'auteur d'abord, et parce que c'est la première œuvre capitale sortie des écrivains de la presse, où l'idée spirite soit carrément

\footnotetext{
9 Kardec écrit dans ce texte: «Nous n'avons pas l'honneur de connaître personnellement l'auteur; nous ne savons quelles sont ses convictions ou ses connaissances touchant le Spiritisme; son ouvrage, qui en est au début, ne permet pas encore d'en voir la conclusion. » (Cité par Tortonese, 1995b, p. 1705)
} 
affirmée, et qu'il a paru dans un moment où il semblait être un démenti jeté au milieu du flot d'attaques dirigées contre cette idée. (Cité par Tortonese, 1995b, p. 1705)

L'auteur de ces articles voit finalement dans Spirite une publication dont la parution s'avère très à propos, sinon capitale, puisqu'elle fait fonction d'arme d'assaut contre les incrédules et les sceptiques qui s'insurgent, au milieu des années 1860, contre le mouvement spirite ${ }^{10}$. La légère hésitation dont il fait néanmoins preuve dans ce constat s'explique sans doute en partie par l'aspect swedenborgien de l'œuvre qui, comme nous l'avons noté, contraste sous plusieurs angles avec les théories spirites.

À côté de ces critiques qui, mutatis mutandis, apprécient le service que Gautier aurait rendu aux sciences occultes, existent bien évidemment d'autres lectures de Spirite marquées au coin du scepticisme et qui mettent plutôt en valeur le talent et la verve poétique de Gautier. Émile Zola, par exemple, écrit dans le journal L'Événement du 16 février 1866 :

[J]e me défie de la foi de Théophile Gautier. Il a une bonhomie ironique qui sent l'incrédulité d'une lieue. Je le soupçonne d'être entré dans l'invisible pour le seul plaisir de décrire à sa guise des horizons imaginaires. [...] Quoi qu'il écrive, Théophile Gautier est toujours écrivain pittoresque et poète original. (1966, t. 10, p. 370-371)

Cette appréciation de Zola est au diapason de celle du Grand Dictionnaire universel du XIXe siècle à l'article portant sur la nouvelle de Gautier, « Spirite » :

\footnotetext{
10 Quelle que soit l'attitude de Gautier à l'égard du swedenborgisme et du spiritisme, le critique a raison d'affirmer que les écrivains qui se prononcent officiellement à ce sujet sont rares. Le cas d'Arthur Conan Doyle fait exception. Voir Prévost (2005).
} 
Les extravagances du spiritisme prennent dans ce livre, merveilleusement écrit[,] des teintes si poétiques et si vaporeuses, qu'on s'y laisse aller comme aux plus séduisantes créations. Ce qu'il y a de plus singulier, c'est que l'auteur, sceptique et matérialiste, ne croyant pas un traître mot des choses surnaturelles auxquelles son imagination prêtait la vie, ait pu si bien entrer dans la peau d'un spirite convaincu; ses conceptions idéales dépassent de beaucoup la fantasmagorie des prétendus adeptes. (Larousse, 1866, t. 14, p. 1017)

Gautier est-il cependant aussi «sceptique et matérialiste » que le prétend ce critique? Ne pourrait-on pas imaginer que Gautier croit aux «choses surnaturelles auxquelles son imagination [prête] la vie »? Doit-on, en fin de compte, se camper dans l'une de ces options au détriment de l'autre? Une réponse à ces questions ne saurait faire abstraction du sous-titre de Spirite: «nouvelle fantastique». C'est justement là que se loge, selon nous, une fructueuse piste de réflexion pour ce qui est de l'attitude de Gautier à l'égard du spiritisme et, d'une manière générale, du paranormal.

Dans ce contexte, notons tout d'abord que Gautier possède une conception particulière du fantastique, laquelle, si elle est influencée par des écrivains tels Achim Arnim, Nodier, Hugo et Nerval, trouve dans l'œuvre d'Ernst Theodor Amadeus Hoffmann une source d'inspiration déterminante. En témoigne toute sa production fantastique. Tantôt ses récits sont hantés par les noms de personnages hoffmannesques - pensons, par exemple, à Crespel et à Antonia, personnages du Violon de Crémone ${ }^{11}$, que l'on mentionne dans le conte Onuphrius ou les vexations fantastiques d'un admirateur d'Hoffmann (Gautier, 1981, p. 74) —; tantôt ils sont truffés d'allusions à l'œuvre du

\footnotetext{
11 Voir Hoffmann, Rat Krespel (1967, t. 2, p. 228-248).
} 
conteur berlinois. Tel est, par exemple, le cas de Spirite où, à la vue des femmes qui assistent à la soirée mondaine chez Madame d'Ymbercourt, le baron de Féroë constate que, pareil $\mathrm{au}$ personnage de Nathaniel qui, dans l'Homme au sable ${ }^{12}$, embrasse une automate, l'on court le risque d'embrasser les femmes sans âme (p.336). Il ne fait donc pas de doute que Gautier est un grand «admirateur » de ce «fantastiqueur »13 qu'est Hoffmann.

Cette admiration se décèle notamment dans les comptes rendus que l'auteur de Spirite rédige au sujet du conteur berlinois. Dans ces mêmes textes, il met aussi en lumière les principaux traits du fantastique hoffmannesque. En voici deux extraits :

La cause de la rapidité du succès d'Hoffmann est assurément là où personne ne l'aurait été chercher. - Elle est dans le sentiment vif et vrai de la nature qui éclate à un si haut degré dans ses compositions les moins explicables. [...] Sa manière de procéder est très logique, et il ne chemine pas au hasard dans les espaces imaginaires, comme on pourrait le croire. [...] [L]e merveilleux d'Hoffmann n'est pas le merveilleux des contes de fées; il a toujours un pied dans le monde réel [...] $]^{14}$. (1883, p. 43-46)

Hoffmann passe pour un poëte fantastique; cependant, jamais réputation ne fut moins méritée, car c'est, au contraire, un réaliste violent. [...] Quand Hoffmann commence un conte, tout va d'abord le plus naturellement du monde; [...] mais bientôt le poêle ronfle avec un son étrange et guttural, le brouillard se condense, l'ombre s'entasse dans les coins, où les chimères commencent à grimacer [...]. Certes, tout cela n'est pas naturel,

\footnotetext{
12 Voir Hoffmann, Der Sandman (1967, t. 2, p. 7-40).

13 Le terme est de Gautier. Il l'utilise dans un texte inédit sur Hoffmann rédigé en 1831 (Spoelberch de Lovenjoul, 1887, t. 1, p. 12).

14 Cet article, intitulé « Les Contes d'Hoffmann », paraît pour la première fois dans la Chronique de Paris, le 14 août 1836.
} 
et nous sommes loin du point de départ; mais quel art infini, quelles savantes préparations et quelle vraisemblance dans l'incroyable! Comme toutes les fibres de l'imagination sont tendues dès le début! malgré la tranquillité apparente du narrateur, comme on devine qu'il va arriver quelque chose! comme, au premier soupir du vent dans le corridor, comme, au premier craquement de la boiserie, on se sent inquiet, ému! [...] et alors vous voilà emporté dans le monde invisible, à la merci du poëte $[s i c]^{15}$. (1858, t. 6, p. 230-231)

Ces passages mettent clairement en relief la place dominante qu'occupe, suivant l'analyse originale de Gautier, la vraisemblance dans le fantastique d'Hoffmann. À vrai dire, l'habileté dans les descriptions préparatoires au surgissement du paranormal que loue Gautier chez Hoffmann est également la sienne, et la vraisemblance est tout aussi prépondérante dans sa propre conception du fantastique. C'est pourquoi, pour l'auteur de Spirite, le terme de fantastique, qui a des origines germaniques (voir Larousse, 1866, t. 8, p. 93-95), se distingue nettement du merveilleux des contes de fées. Selon la définition proposée par Tzvetan Todorov, le merveilleux implique un univers régi par des lois inconnues, nouvelles, c'est-à-dire différentes de celles qui règlent le monde réel communément admis comme tel (1970, p. 46-47). Envisagé dans cette perspective, le récit merveilleux repose inévitablement sur ce que Samuel Taylor Coleridge appelle « la suspension volontaire de l'incrédulité » puisque c'est justement cette suspension, laquelle constitue la «foi poétique ${ }^{16} »(1984$, t. 2 , p. 6), qui

\footnotetext{
15 Cet article paraît sous son titre original, « Odéon : Les contes d'Hoffmann, drame fantastique, de MM. Jules Barbier et Michel Carré », dans La Presse, le 24 mars 1851 (Gautier, 1858, t. 6, p. 230; Spoelberch de Lovenjoul, 1887, t. 1, p. 442).

16 Nous traduisons « willing suspension of disbelief » et « poetic faith ».
} 
ouvre la voie à l'actualisation de tous les possibles lors de la lecture ou de l'écriture d'une œuvre. Non pas que le récit fantastique, telle toute œuvre littéraire, ne dépende pas d'une "foi poétique », loin de là; mais, dans le récit fantastique, cette foi n'est pas absolue, car, contrairement au merveilleux, le fantastique ne se déploie pas à l'écart du monde réel et, partant, il exige une certaine logique émanant de ce même monde. Gautier se prononce explicitement sur cette question :

[D]es imitateurs [d'Hoffmann] [...] ont cru qu'il suffisait d'entasser absurdités sur absurdités et d'écrire au hasard les rêves d'une imagination surexcitée, pour être un conteur fantastique et original; mais il faut dans la fantaisie la plus folle et la plus déréglée une apparence de raison, un prétexte quelconque, un plan, des caractères et une conduite, sans quoi l'œuvre ne sera qu'un plat verbiage [...]. (1883, p. 48)

$\mathrm{Au}$ fond, ce qui importe dans les récits fantastiques de Gautier, c'est que, tout invraisemblables qu'ils soient, ils produisent un effet de réel. De là viennent justement la progression relativement aux apparitions du paranormal dans Spirite et l'effort de décrire fidèlement ce que perçoivent les personnages de cette œuvre. «C'est le positif et le plausible du fantastique », souligne Gautier, «et, à vrai dire, les contes d'Hoffmann devraient plutôt être appelés capricieux ou fantasques, que contes fantastiques » (ibid., p. 46). Quoique le vraisemblable, le positif et le plausible jouent alors un grand rôle dans la conception du fantastique de Gautier ${ }^{17}$, la part de l'imaginaire - comme le signalent les mots «capricieux » et «fantasque» dans les propos cités - n'y est pour autant

17 Comme le fait remarquer Marc Eigeldinger, la conception du fantastique de Gautier s'approche dans cette optique de l'étrange tel que défini par Todorov (Eigeldinger, 1981, p. 20; Todorov, 1976, p. 46-47). 
nullement moindre. Car le souci de produire un effet de réel se conjugue chez Gautier, à l'instar d'Hoffmann, avec les descriptions poussées à l'extrême, démarche qui, en permettant d'explorer tous les coins et recoins de la réalité, aboutit à un éclairage si différent et si insolite du réel que celui-ci se mue en irréel. C'est dire que le «para-normal » chez Gautier devrait être entendu dans l'acception première du terme, c'est-à-dire ce qui existe " à côté du » normal, à travers lui et grâce à lui, bref, ce qui, de manières plus ou moins voilées, coexiste avec lui. À nos yeux, l'aspiration de Gautier à explorer cette concomitance du normal et du paranormal est telle qu'il n'hésite pas à avoir recours aux sciences occultes qui lui sont contemporaines peu importe qu'il s'agisse du magnétisme, du spiritisme ou du swedenborgisme - et qui lui servent précisément de «prétexte», de «plan», dans le dessein d'«emporter [son lectorat] dans le monde invisible ", de lui faire voir et sentir aussi bien le fantastique du réel que le réel du fantastique (voir Voisin, 1980, et Lacroix, 1991, 1992).

Le contexte de la publication de Spirite en dit long sur cette fonction que semblent remplir les sciences occultes dans l'œuvre fantastique de Gautier. Il est intéressant de noter à ce sujet que, à la suite de la parution d'Avatar (1856) et de Jettatura (1856), qui traitent tous les deux, à des degrés divers, du magnétisme (voir Gautier, 1981), Gautier abandonne la publication projetée de Magnétisme, une œuvre qu'il avait envisagée de rédiger d'après une lettre destinée à son éditeur Jules Hetzel en (octobre?) $1856^{18}$ et où germa

\footnotetext{
18 Gautier écrit à Jules Hetzel : « [J]'ai fait depuis Avatar "Paul d'Aspremont ou le Jettatore" de même longueur et même un peu plus long que le susdit. Cette histoire est basée sur la même idée - c'est-à-dire sur l'emploi du fantastique dans la vie réelle. [...] J'ai encore deux contes à faire formant chacun un
} 
vraisemblablement l'idée première de Spirite (voir Montandon et Saminadayar-Perrin, 2003, p. 273). Si Gautier choisit de prendre le spiritisme comme "prétexte », comme " plan», pour sa dernière nouvelle fantastique, c'est sans doute parce que les phénomènes des tables tournantes et de la médiumnité, qui commencent à estomper quelque peu la gloire du magnétisme, sont, vers la fin des années 1850 et dans les années 1860, plus propices à attirer le lecteur dans les aires imaginaires où, « à la merci du poëte », il se voit engagé dans l'exploration de mondes nouveaux créés et recréés par le chevauchement continuel du normal et du paranormal.

\section{Spirite : « Effet magique » ou « effet littéraire »?}

Procédant de la rencontre entre le monde visible et le monde invisible, entre le normal et le paranormal, la narration de Spirite s'élabore de manière logique. En ce sens, l'effacement progressif du scepticisme de Malivert devant les phénomènes surnaturels qu'il éprouve va de pair avec l'évolution de ses facultés médiumniques et le rapprochement de son âme avec celle de Spirite. Il s'ensuit que les principes du spiritisme et du swedenborgisme se voient fidèlement représentés dans cette œuvre de Gautier.

Il ne faudrait pourtant pas en conclure que Gautier cherche à confirmer ou à infirmer la validité de l'une ou l'autre de ces deux sciences occultes, comme l'ont voulu bon nombre de ses contemporains. La réaction positive de la part des 
adeptes de l'occultisme pourrait dériver, d'une part, de l'unicité de l'œuvre - Gautier ayant été considéré comme le premier écrivain à se prononcer ouvertement sur le spiritisme - et, d'autre part, de la vraisemblance et de la plausibilité qui caractérisent la démarche narrative de Spirite. Seulement voilà, vraisemblance et plausibilité ne concourent pas au but que l'on a faussement assigné à cette œuvre : l'engagement. C'est là un objectif auquel Gautier ne paraît nullement avoir songé. « [J]e ne me doutais guère de ce que je faisais », déclare-t-il en commentant l'accueil public de Spirite (1985, t. 9, p.148). À bien y penser, vouloir accoler une valeur utilitariste à une œuvre de Gautier a de quoi étonner. Tout se passe comme si l'on avait complètement oublié le jeune homme au gilet rouge engagé dans la bataille d'Hernani (1830), événement que le grand défenseur de «l'art pour l'art» se rappellera jusqu'au tout dernier jour de sa vie (Ubersfeld, 1992, p. 25); comme si l'on avait mis au placard la célèbre préface de Mademoiselle de Maupin (1835), où Gautier décrie l'utilitarisme dont on voudrait, trop souvent selon lui, charger les artistes et où il déclare : «Il n'y a de vraiment beau que ce qui ne peut servir à rien; tout ce qui est utile est laid [...].» (2004, p. 102) Dès lors, on est en mesure de mieux appréhender l'ébahissement de Gautier devant l'avis de ceux qui jugent que Spirite aurait servi à la défense d'une cause quelconque. En définitive, ce n'est pas un hasard si Gautier qualifie une telle opinion, non sans un brin d'ironie, d'« effet magique » $\left(1985\right.$, t. 9, p. 147) ${ }^{19}$.

Loin de viser pareil coup de magie débouchant sur un engagement social en faveur de l'occultisme, les principes de

19 Voir la lettre de Gautier adressée à Carlotta Grisi citée plus haut. 
vraisemblance et de plausibilité mis en œuvre dans Spirite ont plutôt en vue " un effet littéraire » (ibid.), d'autant plus qu'ils appartiennent, comme nous avons pu le constater, à la conception du fantastique de Gautier. Tout en étant centrée sur un effet de réel, cette conception repose sur l'exploration des rapports symbiotiques entre le normal et le paranormal. C'est justement grâce à ce jeu agile sur l'interstice, qui signifie transgression du réel et de l'irréel, que le fantastique de Gautier répond parfaitement à sa réflexion sur la liberté de l'art. Car le jeu sur l'interstice, potentiellement éternel, anime par principe l'imagination de l'artiste, lui permettant ainsi de s'envoler sans cesse vers des mondes autres, plus beaux, plus sublimes, plus idéals, mondes qui, à leur tour, prennent vie dans les œuvres et qui, en se joignant au monde réel, contribuent à l'enrichir en l'embellissant.

Cela étant, nous pouvons maintenant revenir sur notre question de départ: qu'en est-il d'un portrait de Gautier en spirite? À ce sujet, Gautier lui-même aurait affirmé à Émile Bergerat, qui l'a interrogé sur sa croyance relativement au spiritisme: «Non, je n'y crois plus, [...] mais j'y ai cru en écrivant le livre [Spirite]» (1996, p. 169). Compte tenu de la prise de position artistique de Gautier, cette croyance ne peut guère être rapprochée de celle d'un adepte zélé ou d'un initié engagé. Tout au contraire. Si l'auteur de Spirite se dit spirite et qu'il croit en son œuvre, c'est parce que le monde éthéré dans lequel l'emporte l'élan poétique de la création jouit d'une vérité propre, une vérité artistique, qui, pour l'esthète raffiné, en arrive à supplanter, pendant quelque temps, la vérité du monde réel. Nous faire entrevoir l'éclat, sentir le zéphyr et humer le parfum émanant du vaste empyrée, c'est là l'« effet littéraire » 
ultime, aussi éphémère soit-il, vers lequel tend la dernière nouvelle fantastique de Gautier.

\section{Bibliographie}

BERGERAT, Émile. 1996 [1879], Théophile Gautier. Entretiens, souvenirs et correspondance, Paris, L'Harmattan, coll. « Les introuvables ».

BRETON, André. 2005 [1924 et 1930], Manifestes du surréalisme, Paris, Gallimard, coll. «Folio/Essais ».

COLERIDGE, Samuel Taylor. 1984, Biographia Literaria or Biographical Sketches of My Literary Life and Opinions, James Engell et W. Jackson Bate (éd.), Princeton, Princeton University Press, « Bollingen » Series.

DASH, comtesse [vicomtesse Poillouë de Saint-Mars]. 1886, Mémoires des autres, Paris, Librairie illustrée.

EDELMAN, Nicole. 1995, Voyantes, guérisseuses et visionnaires en France (1785-1914), Paris, Albin Michel, coll. « Bibliothèque Albin Michel. Histoire ».

EIGELDINGER, Marc. 1981, "Introduction », dans Théophile Gautier, Récits fantastiques, M. Eigeldinger (éd.), Paris, Flammarion, p. 15-42.

ELLENBERGER, Henri F. 1970, The Discovery of the Unconscious. The History and Evolution of Dynamic Psychiatry, New York, Basic Books Inc.

GAUTIER, Judith. 1903 [?], Le second rang du collier, Paris, Félix Juven. 
GAUTIER, Théophile. 1858, Histoire de l'art dramatique en France depuis vingt-cinq ans, Paris, Hetzel;

-. 1883, Souvenirs de théâtre, d'art et de critique, Paris, G. Charpentier;

—. 1981, Récits fantastiques, Marc Eigeldinger (éd.), Paris, Flammarion;

—. 1985, Correspondance générale, Claudine Lacoste-Veysseyre (éd.), Genève, Droz;

-. 2003, Spirite, dans CEuvres complètes, t. 5, Alain Montandon et Corinne Saminadayar-Perrin (éd.), Paris, Honoré Champion;

—. 2004, Mademoiselle de Maupin, dans CEuvres complètes, t. 1, Anne Geisler-Szmulewicz (éd.), Paris, Honoré Champion.

HoffmanN, Ernst Theodor Amadeus. 1967, Werke, Herbert Kraft et Manfred Wacker (éd.), Frankfurt am Main, Insel Verlag.

KARDEC, Allan. 1863 [1861], Le Livre des médiums, 6e éd., Paris, Didier et Cie;

—. 1867 [1857], Le Livre des esprits, 15e éd., Paris, Didier et Cie.

LACRoIX, Jean. 1991, «Du "mensonge romantique" à la "fabuleuse réalité". Traitement du réel dans le récit ambigu de Théophile Gautier (I) », Synthesis, vol. 18, p. 77-81;

—. 1992, « Du "mensonge romantique" à la "fabuleuse réalité". Traitement du réel dans le récit ambigu de Théophile Gautier (II) », Synthesis, vol. 19, p. 95-105.

LAROUSSE, Pierre. 1866, Grand Dictionnaire universel du XIXe siècle, Paris, Larousse et Boyer.

LEFEBVRE, Anne-Marie. 1993, «Théophile Gautier et les spirites et illuminés de son temps », Bulletin de la Société Théophile Gautier, vol. 15, no 2, p. 291-322. 
—. 1996, «De Séraphîta à Spirite : le génie et l'ange », L'Année balzacienne, vol. 17, p. 245-267.

MonTANDON, Alain et Corinne SAMINADAYAR-PERRIN. 2003, "Introduction. Spirite, ou l'amour plus fort que la mort", dans Théophile Gautier, Spirite, dans OEuvres complètes, t. 5, Alain Montandon et Corinne Saminadayar-Perrin (éd.), Paris, Honoré Champion, p. 273-315.

PrÉvost, Maxime. 2003, «Les Mystères de Jersey. Représentations de Hugo en spirite », dans Maxime Prévost et Yan Hamel (dir.), Victor Hugo (2003-1802). Images et transfigurations, Montréal, Fides, p. 29-41;

—. 2005, «L'Expérimentation spirite de deux pères: Victor Hugo et Arthur Conan Doyle », Frontières, vol. 18, no 1 , automne, p. 27-32;

-. 2006, «Les Savants et les sachants. L'engagement scientifique chez Balzac et Dumas », Les Cahiers du XIXe siècle, no 1 , p. 97-113.

RICARD, Jean Joseph Adolphe. 1843, Lettres d'un magnétiseur, Paris, Chez l'auteur.

SPOElBERCH DE LOVENJOUL, vicomte de [Charles de Lovenjoul]. 1887, Histoire des cuvres de Théophile Gautier, Paris, G. Charpentier et Cie.

SWEDENBORG, Emmanuel. 1973 [1758], Le Ciel et ses merveilles et l'enfer d'après ce qui a été entendu et vu, Paris, Cercle Swedenborg.

Todorov, Tzvetan. 1976 [1970], Introduction à la littérature fantastique, Paris, Seuil, coll. « Points ».

TORTONESE, Paolo. 1995a, «Chronologie », dans Théophile Gautier, CEuvres. Choix de romans et de contes, Paolo Tortonese (éd.), Paris, Robert Laffont, coll. «Bouquins", p. XXXIII-LII. 
—. 1995b, "Spirite. Notice », dans Théophile Gautier, CEuvres. Choix de romans et de contes, Paolo Tortonese (éd.), Paris, Robert Laffont, coll. «Bouquins », p. 1701-1709.

UBERSFELD, Anne. 1992, Théophile Gautier, Paris, Stock.

VoISIN, Marcel. 1980, «L'Insolite quotidien dans l'œuvre en prose de Théophile Gautier», Cahiers de l'Association internationale des études françaises, no 32, mai, p. 163-178.

ZolA, Émile. 1966, CEuvres complètes, Henri Mitterand (éd.), Paris, Cercle du livre précieux.

\title{
Résumé
}

Théophile Gautier puise dans la vague des sciences occultes qui déferle à son époque la matière de Spirite, œuvre qui connaît une réception ambivalente. Alors que certains la considèrent comme une défense et illustration de l'occultisme, d'autres, plus sceptiques, en chantent plutôt le souffle poétique. Cet article propose une réflexion sur l'attitude de Gautier à l'égard des sciences occultes en particulier et du paranormal en général. On cherchera notamment à montrer que, si le paranormal occupe une place importante dans l'œuvre de Gautier, l'auteur se présente moins comme un initié engagé que comme un esthète raffiné.

\begin{abstract}
Théophile Gautier finds in the sweeping wave of occult sciences of his time a source of inspiration for Spirite, a work which divides public opinion. While it is seen by some as a means of defence for occultism, it is celebrated by others, who are more
\end{abstract}


sceptical, rather for its poetic qualities. This article focuses on Gautier's attitude towards occult sciences in particular and the paranormal in general. We will, more specifically, aim to show that, even though the paranormal plays an important part in Gautier's works, the author appears less to be a committed initiate than a refined aesthete. 